\title{
Tomografía Computarizada de Haz Cónico como Instru- mento Complementario de Diagnóstico y Planeamiento Quirúrgico de Quiste Dentígero: Reporte de un Caso
}

\author{
Cone-Beam Computed Tomography as Complementary Tool in Diagnosis \\ and Surgical Planning of Dentigerous Cyst: Case Report
}

\begin{abstract}
Maria Augusta Portella Guedes Visconti*; Rafael Binato Junqueira*; Francielle Silvestre Verner*; Abílio Augusto Sobrinho Rodrigues*; Karina Lopes Devito* \& Renato Francisco Visconti Filho*
\end{abstract}

VISCONTI, M. A. P. G.; JUNQUEIRA, R. B.; VERNER, F. S.; RODRIGUES, A. A. S.; DEVITO, K. L. \& VISCONTI FILHO, R. F. Tomografía computarizada de haz cónico como instrumento complementario de diagnóstico y planeamiento quirúrgico de quiste dentígero: Reporte de un caso. Int. J. Odontostomat., 8(1):85-91, 2014.

RESUMEN: El quiste dentígero es una de las patologías óseas que más frecuentes en el maxilar y la mandíbula. Se evidencia radiográficamente como una imagen radiolúcida y unilocular en la mayoría de los casos. Generalmente es detectado en exámenes de rutina o cuando se investiga la presencia de dientes no erupcionados. El quiste dentígero aparece principalmente en las tres primeras décadas de vida, con crecimiento lento y asintomático. Los terceros molares,así como dientes supernumerarios, pueden estar relacionados con su formación, sin embargo, su etiopatología no es totalmente conocida. La descompresión, marsupialización y la enucleación son las principales formas de tratamiento, pero algunos criterios deben ser considerados, como tamaño del quiste, edad del paciente, proximidad con estructuras anatómicas nobles e importancia clínica del diente involucrado. Para que se establezca un correcto diagnóstico y plan de tratamiento es esencial la utilización de exámenes complementarios de calidad, siendo, actualmente, la tomografía computarizada de haz cónico el más completo y preciso método de diagnóstico por imagen empleado en estas lesiones. El objetivo de este estudio fue resaltar, por medio de la presentación de un caso clínico, la importancia de la tomografía computarizada de haz cónico en el diagnóstico y planeamiento quirúrgico de un quiste dentígero asociado a un diente no erupcionado.

PALABRASCLAVE:Diagnóstico, imagen tridimensional, quiste dentígero.

\section{INTRODUCCIÓN}

Elquiste dentígero (QD) o folicular es el segundo tipo máscomún de quiste odontogénico (Sette-Dias et al., 2008). La histogénesisexacta de formación y expansión de esta patologíapermanece incierta, aunque fue sugerido que el origen es la acumulaciónde líquido entre el epitelio reducido del órgano del esmalte y lacorona subyacente (Silva Júnior et al., 2006).

Ocurre en los huesos maxilar o mandibular con una frecuencia entre $14 \%$ y $20 \%$, sólo después de los quistes periapicales. Generalmente es unilocular y de mayor ocurrencia en la mandíbula y en el sexo masculino (Safira et al., 2009; Vaz et al., 2010). Son siempre radiolúcidos y más comúnmente uniloculares, aunque las grandes lesiones pueden presentar un padrón multilocular (Vaz et al.).
Clínicamente el QD es una lesión asintomática de crecimiento lento, lo que dificulta el diagnóstico clínico. Cuando se establecen como grandes lesiones pueden producir alteración en el contorno facial (asimetría), debido a la expansión de las corticales y otras complicaciones clínicas como desplazamiento de dientes, erupción ectópica, impactación dentaria, reabsorción de las raices de los dientes adyacentes y hasta malignización de la lesión (Sette-Dias et al.; Mezzomo et al., 2009).

Este quiste es descubierto, usualmente en exámenes radiográficos realizados con otra finalidad, especialmente al investigarse la no erupción de un diente permanente, ya que la remoción de dientes no erupcionados es recomendable como método de pre- 
venir el surgimiento de quistes o tumores odontogénico (Martins Filho et al., 2008; Sumida et al., 2009; Kim \& Mun, 2013).

Como esta lesión desencadenaría diversas alteraciones morfológicas es necesario disponer de exámenes complementarios de calidad para el diagnóstico correcto y mejor planeamiento del tratamiento. Convencionalmente las radiografías panorámicas son utilizadas para establecer padrones de identificación y planeamiento para la remoción de las lesiones radiolúcidas que suceden en los maxilares (Raitz et al., 2009).

Como el QD se presenta comúnmente asociado a terceros molares inferiores no erupcionados, es de fundamental importancia que sea de conocimiento del profesional la real localización en relación al nervio alveolar inferior. El canal de la mandíbula en esta región se puede presentar en diversas posiciones anatómicas, lo que limita la indicación de las radiografías panorámicas para su correcta determinación. A fin de evitarse accidentes en el acto quirúrgico, como lesiones permanentes al paquete vasculonervioso alveolar inferior, es imprescindible la utilización de un recurso diagnóstico que permita la visualización tridimensional de toda la región involucrada.

Actualmente la tomografía computarizada de haz cónico $(\mathrm{TCHC})$ viene siendo utilizada en diversas áreas de la Odontología para garantizar un diagnóstico preciso y correcto plan de tratamiento (Santos et al., 2009). Posee como ventaja alta definición de las imágenes, una dosis de radiación de 1/6 de la liberada por la tomografía computarizada tradicional, costo del equipo muy reducido, rápida adquisición de la imagen con mayor conforto del paciente, que no necesita de un ambiente hospitalario (Fernandes et al., 2009). Además de esto, a partir de un corte axial de TCHC es posible obtener reconstrucciones coronarias, sagitales, cortes perpendiculares, reconstrucciones en 3D e imágenes convencionales bidimensionales. Estos recursos posibilitan mayor precisión diagnóstica, mejorar la planificación y el tratamiento propuesto, siendo el examen indicado en patologías como el QD.

El método de tratamiento más común para esta patología consiste en la remoción del quiste y del diente asociado, pudiendo también ser utilizada la técnica de marsupialización o tapizamiento en casos específicos (Silva Júnior et al.; Yücel et al., 2013).

El objetivo del presente estudio fue relatar, por medio de la presentación de un caso clínico, la importancia de la utilización de la TCHC en el diagnóstico y planeamiento quirúrgico de un quiste dentígero asociado a un diente no erupcionado.

\section{RELATO DEL CASO}

Paciente de 36 años de edad, de sexo femenino, de piel blanca, se presento en la clínica de Cirugía Maxilofacial III de la Facultad de Odontología de la Universidad Federal de Juiz de Fora, relatando la presencia de patología en la mandíbula, evidenciada por un examen radiográfico panorámico que fue ejecutado con la finalidad de evaluar dientes retenidos. En el referido examen se observaba la presencia de retención de los dientes 18, 28, 38 y 48. Asociado al diente 48 , que estaba en posición horizontal, se verificó la presencia de una extensa lesión radiolúcida, unilocular, de contornos definidos por un halo radiopaco, con

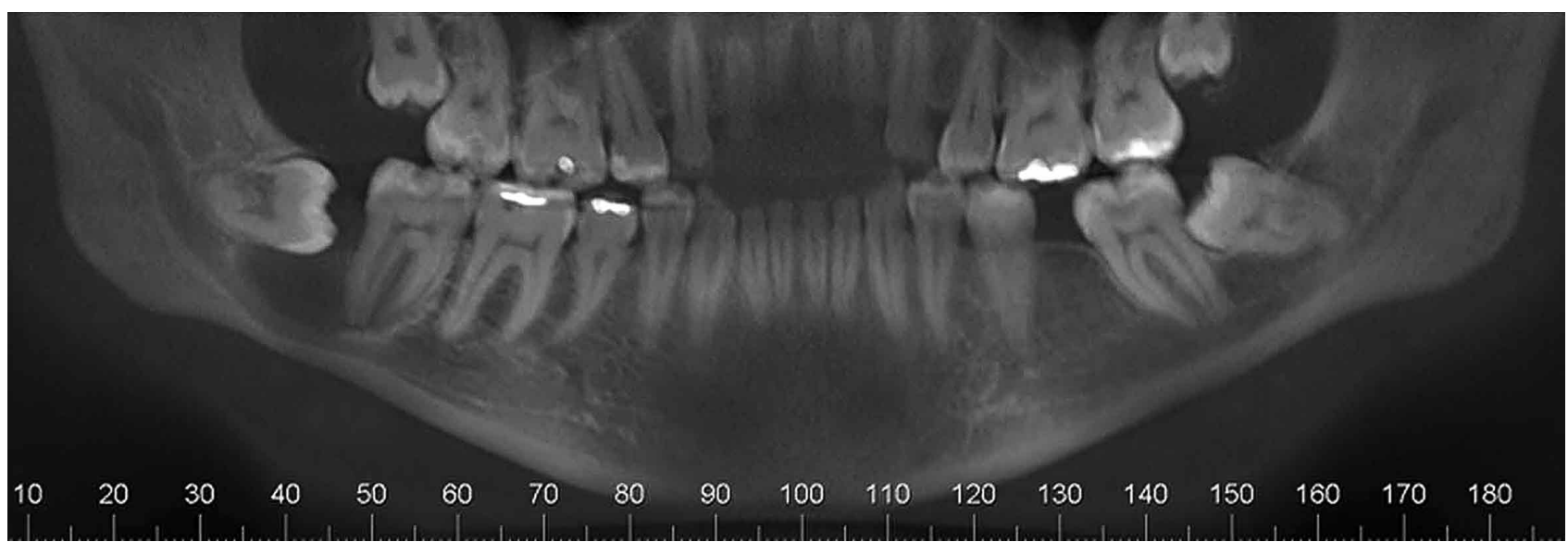

Fig. 1. Reconstrucción panorámica del examen de TCHC evidenciando los mismos aspectos verificados en la radiografía panorámica convencional. 
aproximadamente $2 \mathrm{~cm}$ en su mayor diámetro, extendiéndose anteriormente hasta el diente 47 , con aparente participación de su raíz distal (Fig. 1).

El trayecto del canal de la mandíbula se presentaba desplazado inferiormente hasta el borde inferior con nítida alteración de su trayecto normal. Pareciendo haber discreta interrupción de la cortical en la porción más coronaria del contorno de la lesión, próximo a distal del diente 47 (Fig. 1).

Al examen clínico se observaba un discreto aumento de volumen a distal del diente 47 , sin embargo, la paciente no presentaba ninguna sintomatología. La mucosa de recubrimiento de la región del diente 48 se presentaba sin alteraciones. A la palpación se observó la presencia de una fluctuación en distal del diente 47, evidenciando la ausencia de la cortical ósea superior. Fue entonces realizada la punzo aspiración de esta región, determinando la naturaleza quística de la lesión.

El examen de TCHC posibilitó correcta evaluación de la relación de la lesión con el paquete vasculonervioso alveolar inferior y su cercanía con las raices del diente 47. En este examen fue verificada la estrecha relación de la lesión con el canal mandibular, desplazándolo a la parte inferior del cuerpo mandibular con abultamiento de la cortical lingual e interrupción del contorno de la lesión en la porción coronal, próxima a la parte distal del diente 47. En los cortes axiales, de las piezas 35 a la 48 , se puede evidenciar la ausencia de la cortical superior del canal de la mandíbula lo que colocaba al paquete neurovascular alveolar inferior en contacto directo con la cápsula de la lesión (Fig. 2).
La raíz distal del diente 47 se presentaba envuelta por la lesión, sin involucrar su cortical alveolar. La raíz del diente 48 se extendía hasta la rama mandibular, próximo al canal de la mandíbula, sin embargo, se mostraba en contacto directo con su cortical superior.

El tratamiento quirúrgico ejecutado fue la exodoncia del diente 48 con enucleación del quiste intraóseo por medio del cuidadoso curetaje, debido a la proximidad de las estructuras anatómicas ya mencionadas. El procedimiento fue realizado con anestesia local (bloqueo de los nervios alveolar inferior, lingual y bucal por la técnica de Gow-Gates). El anestésico utilizado fue la lidocaína al $2 \%$ con adrenalina a $1: 100.000$.

El acceso a la lesión se obtuvo por colgajo mucoperiostico total a partir de una incisión lineal a lo largo de la cresta alveolar en la región del diente 48, que se extendió al surco gingival en el cuello anatómico del diente 47 y una incisión relajante en mesial hasta la proximidad del fondo de surco vestibular. Durante el desplazamiento del colgajo se comprobó la interrupción de la cortical mandibular en la superficie del reborde distal del diente 47 (Fig. 3).

Fue realizada la osteotomía para la extensión del acceso quirúrgico al diente y la lesión, con fresas esféricas número 8 con irrigación con suero fisiológico frio. La lesión se presentaba con la capsula íntegra, pero no con mucho espesor. Esta fue incisionada y el liquido aspirado para la visualización del diente retenido, que fue, entonces, removido conjuntamente con la lesión cureteada (Fig. 4).

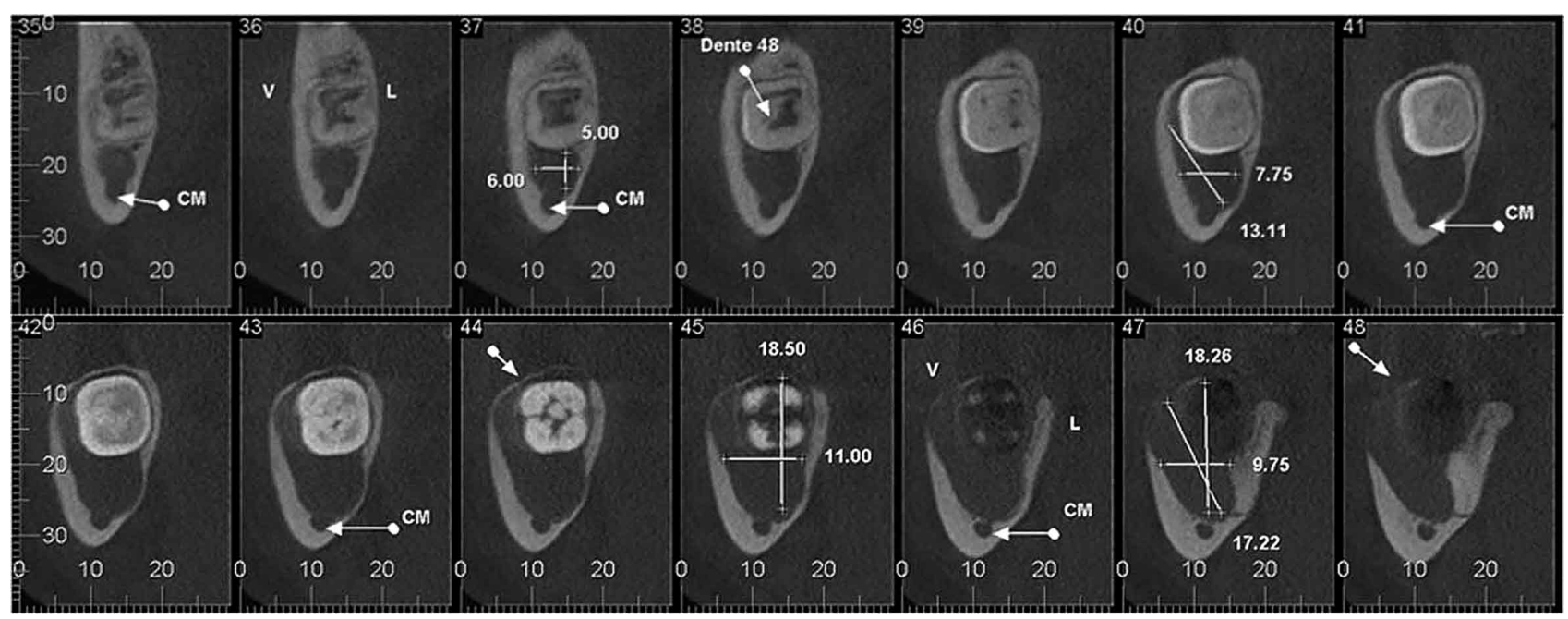

Fig. 2. Reconstrucciones oblicuas (cortes 35 a 48) del examen de TCHC evidenciando la ausencia de la cortical superior del canal de la mandíbula. 


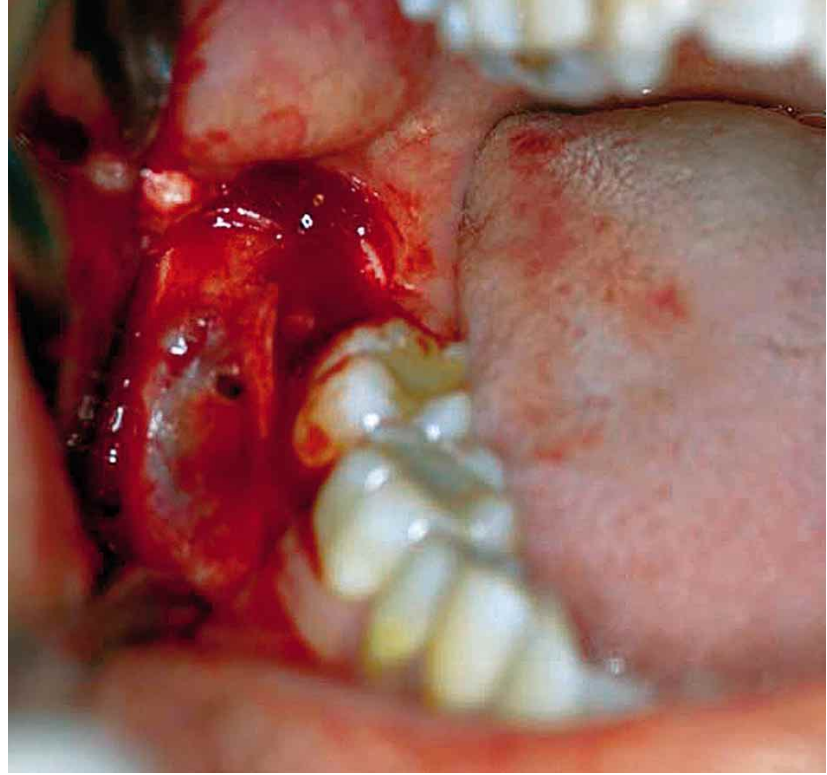

Fig. 3. Acceso quirúrgico evidenciando la cápsula quística.

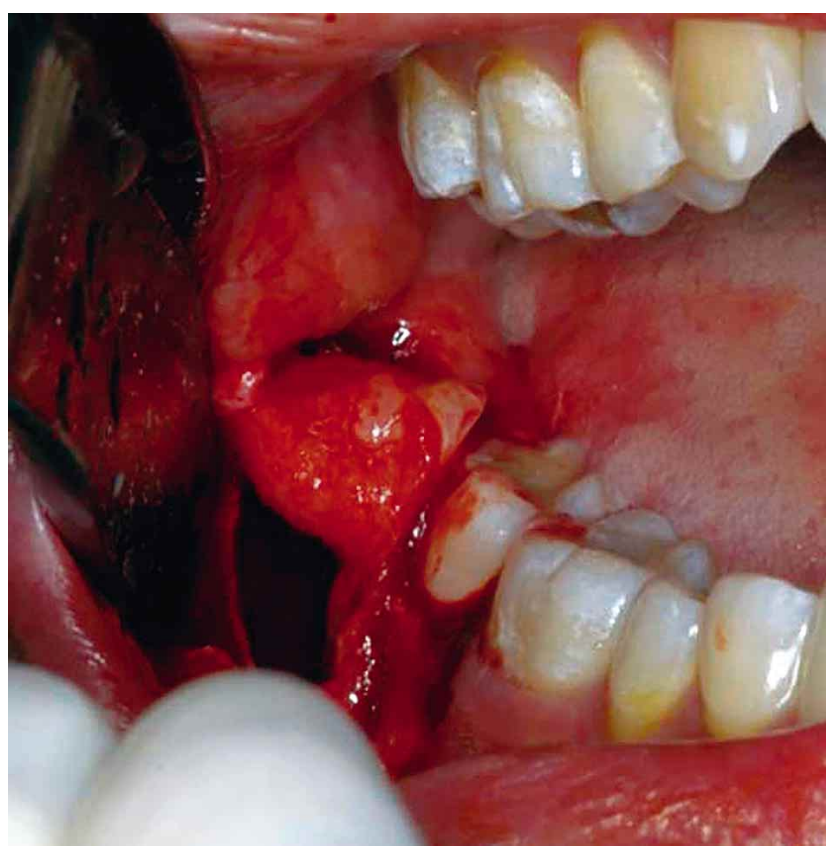

Fig. 4. Enucleación total de la lesión con remoción del diente retenido.

Después de la remoción de la lesión se pudo observar directamente el paquete vasculonervioso alveolar inferior en el fondo de la cavidad ósea, ya que el canal de la mandíbula no presentaba cortical superior, por una extensión de aproximadamente $1 \mathrm{~cm}$. La herida quirúrgica fue suturada con puntos simples aislados e hilo de seda negra trenzada 3.0.
Se prescribió analgésico, antiinflamatorio y antibiótico en el posoperatorio y enjuague bucal suave hasta que la sutura fuera removida, lo que sucedió después de una semana. La pieza quirúrgica fue enviada para la histopatología (Fig. 5) siendo el diagnóstico de quiste dentígero.

En los controles posoperatorios (seis meses), la paciente no tuvo síntomas clínicos ni radiográficos de recidiva de la lesión.

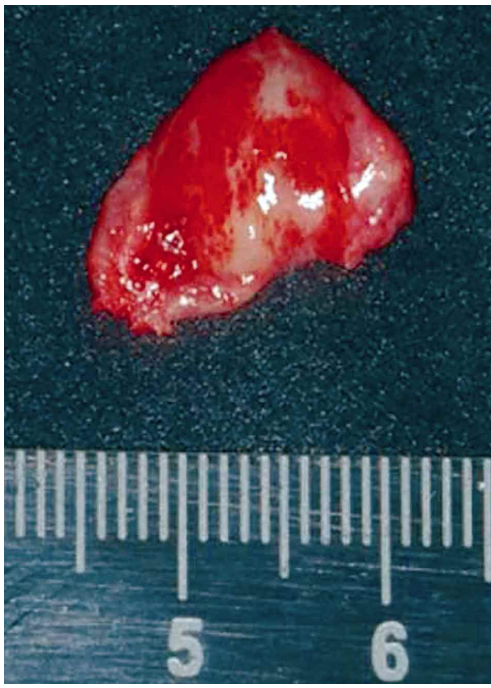

Fig. 5. Pieza quirúrgica después de la enucleación.

\section{DISCUSIÓN}

EI QD es considerado el segundo tipo de quiste odontogénico más prevalente en los maxilares, comprendiendo cerca del $20 \%$ de todos los quistes epiteliales (Godoy et al., 2002; Silva Júnior et al.; Safira et al.).

La patogénesis de este quiste todavía es bastante discutida. Algunos autores afirmaron que se desarrolla precozmente en la odontogénesis, generado por la degeneración del retículo estrellado del órgano del esmalte (Silveira et al., 2009; Slootwek, 2010). Otra posibilidad es el desarrollo del quiste después de la completa formación de la corona, cuando ocurre el acúmulo de liquido entre la corona y el epitelio reducido del esmalte (Godoy et al.).

Los dientes más frecuentemente involucrados son los terceros molares inferiores y los caninos superiores, siendo que los molares representan $75 \%$ de los casos (Osawa et al., 2008). El caso clínico descrito resaltó la 
VISCONTI, M. A. P. G.; JUNQUEIRA, R. B.; VERNER, F. S.; RODRIGUES, A. A. S.; DEVITO, K. L. \& VISCONTI FILHO, R. F. Tomografía computarizada de haz cónico como instrumento complementario de diagnóstico y planeamiento quirúrgico de quiste dentígero: Reporte de un caso. Int. J. Odontostomat., 8(1):85-91, 2014.

relación de los terceros molares no erupcionados con la lesión radiolúcida encontrada en la mandíbula.

A pesar de la controversia, algunos autores sugieren todavía que los quistes foliculares poseen potencialidad definida para transformarse en tumores, especialmente ameloblastomas, lo que reitera la importancia de la enucleación total de la lesión (Mezzomo et al.).

Clásicamente, el tratamiento quirúrgico del QD es la enucleación de la lesión y remoción del diente involucrado (Vaz et al.). Al igual que en el caso presentado, este tratamiento puede ser favorable en los casos por ejemplo de no erupción de terceros molares (Cankurtaran et al., 2010). Por otro lado, podría ocurrir la perdida de varios dientes o lesión importante de estructuras anatómicas adyacentes (Yulis et al., 2007).

Basándose del hecho de que el QD es una lesión benigna, algunos factores o criterios de evaluación pueden determinar que opción de tratamiento sería la más adecuada en cada caso (Ramesh \& Pabla, 2009). El tamaño del quiste es un factor importante a ser considerado en el planeamiento del tratamiento. Quistes pequeños pueden ser fácilmente enucleados y sometidos a un examen histopatológico (biopsia excisional), mientras se preserva el diente afectado (Yulis et al.). Sin embargo, en los casos donde hay expansión de corticales, hecho encontrado en el caso descrito, el desplazamiento mucoperiostal necesario para el tratamiento por enucleación puede ser indicado, pues la cortical adelgazada difícilmente se mantendrá viable después del acto quirúrgico (Sumida et al.).

En el caso clínico presentado fue posible identificar adelgazamiento de la cortical mandibular así como desplazamiento del trayecto del canal mandibular en función de la lesión y sin embargo, el tratamiento de elección fue la enucleación completa de la misma con la extracción del diente afectado.

La realización de exámenes complementares de calidad es imprescindible para el establecimiento de un correcto planeamiento quirúrgico, evitándose complicaciones futuras. En el planeamiento de cirugías de terceros molares la radiografía panorámica de los maxilares es la técnica imaginológica más utilizada en el mundo y puede, a veces, servir de parámetro para averiguar el grado de dificultad de la cirugía, dependiendo de la morfología y posición del tercer molar y su relación con estructuras anatómicas adyacentes y riesgos quirúrgicos, particularmente relacionados a la proximidad de la lesión al nervio alveolar inferior (Raitz et al.; Santos et al.). Sin embargo, las radiografía panorámica es un tipo de examen basado en una imagen bidimensional, lo cual no permite la real observación de toda la dimensión de la lesión, al igual que sus relaciones con las demás estructuras adyacentes (Junqueira et al., 2011).

Según Silveira et al., la diferenciación radiográfica puede ser difícil en los casos en que se ve afectado el diente rodeado de la lesión se presenta impactado. De esta forma, el quiste dentígero puede estar desplazado lateralmente, debido a una expansión.

Debido a las limitaciones impuestas por las imágenes bidimensionales, la TCHC viene siendo establecida como el examen más completo y preciso para Odontología (Fernandes et al.), siendo útil en diversas áreas, especialmente con respecto a los planes quirúrgicos donde hay riesgo de lesiones a las estructuras nobles anatómicas, tales como el nervio alveolar inferior (Guttenberg, 2008; White, 2008). Los exámenes de TCHC permiten reconstrucciones en tres planos distintos, como son: el coronal, sagital y axial. Además las reconstrucciones orto radiales son fáciles de entender e interpretar, posibilitando una clara observación del lugar exacto de la lesión y su relación de contigüidad con las estructuras adyacentes.

La paciente del caso en cuestión fue sometida a exámenes de TCHC para la mejor evaluación de la relación de la lesión con el paquete vasculonervioso alveolar inferior y con las raíces del diente adyacente. El examen fue de suma importancia en la orientación del cirujano en cuanto a la proximidad con tales estructuras, permitiendo que la enucleación de la lesión y la remoción del diente retenido involucrado puedan ser realizadas sin complicaciones 0 secuelas posoperatorios, comunes en casos semejantes.

Es de extrema importancia para el correcto planeamiento y ejecución del tratamiento de lesiones intraóseas, que el cirujano disponga de exámenes complementares imaginológicos que permitan una amplia y verdadera visualización de la lesión y su correlación con estructuras anatómicas vecinas.

La TCHC es un examen de fácil obtención, costo accesible, que permite la clara visualización tridimensional de las lesiones intraóseas, lo que facilita el planeamiento y la ejecución del tratamiento quirúrgico propuesto, evitando complicaciones y secuelas en el acto operatorio y en el posoperatorio. 
VISCONTI, M. A. P. G.; JUNQUEIRA, R. B.; VERNER, F. S.; RODRIGUES, A. A. S.; DEVITO, K. L. \& VISCONTI FILHO, R. F. Tomografía computarizada de haz cónico como instrumento complementario de diagnóstico y planeamiento quirúrgico de quiste dentígero: Reporte de un caso. Int. J. Odontostomat., 8(1):85-91, 2014.

VISCONTI, M. A. P. G.; JUNQUEIRA, R. B.; VERNER, F. S.; RODRIGUES, A. A. S.; DEVITO, K. L. \& VISCONTI FILHO, R. F. Cone-Beam computed tomography as complementary tool in diagnosis and surgical planning of dentigerous cyst: Case report. Int. J. Odontostomat., 8(1):85-91, 2014.

ABSTRACT: The dentigerous cyst is one of the most frequently found in the jaws. They present themselves radiographically as radiolucent images and more commonly unilocular. They are usually observed at routine checkups or when investigating the presence of unerupted teeth. The dentigerous cyst occurs mainly in the first three decades of life, their growth is slow and asymptomatic. The third molars, as well as supernumerary teeth may be involved with the formation of a dentigerous cyst, but its pathogenesis is still not fully known. Decompression, marsupialization and enucleation are the main forms of treatment, but some criteria must be considered for the treatment plan, such as cyst size, age, proximity to noble anatomical structures and clinical importance of the tooth involved. In order to establish a correct diagnosis and treatment plan is essential to make use of complementary exams and now a days the cone beam computed tomography is the most complete and accurate method in diagnostic imaging used in these situations. Therefore, the aim of this study was to highlight, through the presentation of a clinical case, the importance of cone beam computed tomography in the diagnosis and treatment planning of a dentigerous cyst.

KEY WORDS: dentigerous cyst, diagnosis, three-dimensional imaging.

\section{REFERENCIAS BIBLIOGRÁFICAS}

Cankurtaran, C. Z.; Branstetter, B. F. 4th; Chiosea, S. I. \& Barnes, E. L. Best cases from the AFIP: ameloblastoma and dentigerous cyst associated with impacted mandibular third molar tooth. Radiographics, 30(5):1415-20, 2010.

Fernandes, L. M. P. S. R.; Rubira-Bullen, I. R. F. \& Capelozza, A. L. A. Tomografia computadorizada cone beam: aplicações deste novo recurso para o diagnóstico por imagens. Rev. Odontol. UNESP, 38(Esp.):3844, 2009.

Godoy, G. P.; Lins, R. D. A. U.; Medeiros, K. M. S.; Simas Neto, L. M. \& Figueiredo, C. R. L. V. Estudo epidemiológico e avaliação histomorfológica de 108 casos de cisto dentígero. Odontol. Clín. Cient., 1(1):4751, 2002.

Guttenberg, S. A. Oral and maxillofacial pathology in three dimensions. Dent. Clin. North Am., 52(4):843-73, 2008.

Kim, K. S. \& Mun, S. K. Extensive dentigerous cyst associated with a mesiodens: CT findings. Ear Nose Throat J., 92(8):E6-8, 2013.

Junqueira, R. B.; Verner, F. S.; Vilela, E. M.; Devito, K. L.; Chaves, M. G. A. M. \& Carmo A. M. R. Tomografia computadorizada de feixe cônico como instrumento complementar de diagnóstico e planejamento cirúrgico de cisto radicular: relato de um caso clínico. Rev. Odontol. UNESP, 40(6):338-43, 2011.

Martins Filho, P. R. S.; Piva, M. R.; Santos, T. S.; Silva L. C. F. \& Souza, L. B. Avaliação da prevalência de patologias em terceiros molares inclusos. Rev. Cir. Traumatol. Buco-Maxilo-Fac., 8(3):41-8, 2008.
Mezzomo, F.; Dall'Igna, C.; Chevarria, M.; Maahs, M. \& Burzlaff, J. B. Conseqüências ortodônticas do cisto dentígero. Ortodon. Gaúch., 9(1):52-60, 2005.

Osawa, H. K.; Takahashi, A.; Zardo, M.; Gonçalves, R. C. G.; Martins, L. D. \& Felipini, R. C. Cisto dentígero associado a terceiro molar inferior: acesso extra-bucal. Rev. Odontol. UNESP, 37:54, 2008.

Raitz, R.; Assunção Júnior, J. N.; Correa, L. \& FenyoPereira, M. Parameters in panoramic radiography for differentiation of radiolucent lesions. J. Appl. Oral Sci., 17(5):381-7, 2009.

Ramesh, A. \& Pabla, T. Incidental findings on dental radiographs: dentigerous cyst. J. Mass. Dent. Soc., 58(2):42, 2009.

Safira, L. C.; Paim, J. M.; Carneiro Jr., B.; Queiroz, C. S.; Oliveira, T. B.; Ramalho, L. P. \& Sarmento, V. A. Cisto dentígero em mandíbula: relato de caso clínico. Rev. Ciênc. Méd. Biol., 8(2):225-9, 2009.

Santos, T. S.; Cordeiro-Neto, J. F.; Raimundo, R. C.; Frazão, M. \& Gomes, A. C. A. Relação topográfica entre o canal mandibular e o terceiro molar inferior em tomografias de feixe volumétrico. Rev. Cir. Traumatol. Buco-Maxilo-Fac., 9(3):79-88, 2009.

Sette-Dias, A. C.; Abdo, E. N.; Mesquita, R. A.; Dutra, C. E. A. \& Norman-Ferreira, L. C. Cisto dentígero seqüencial: relato de caso. ROBRAC, 17(44):133-7, 2008.

Silva Júnior, N. A.; Weingaertner, E. \& Aguiar, R. C. Cisto dentígero em mandíbula associado a coroa de dois dentes permanentes. RGO, 54(2):157-60, 2006. 
VISCONTI, M. A. P. G.; JUNQUEIRA, R. B.; VERNER, F. S.; RODRIGUES, A. A. S.; DEVITO, K. L. \& VISCONTI FILHO, R. F. Tomografía computarizada de haz cónico como instrumento complementario de diagnóstico y planeamiento quirúrgico de quiste dentígero: Reporte de un caso. Int. J. Odontostomat., 8(1):85-91, 2014.

Silveira, V. A. S.; Pereira, A. C.; Amadei, S. U.; Carmo, E. D. \& Carvalho, Y. R. Cisto dentígero inflamatório relacionado a dente permanente: considerações etiopatológicas. Rev. Odontol. UNESP, 38(3):143-7, 2009.

Slootweg, P. J. Bone diseases of the jaws. Int. J. Dent., 2010:702314, 2010.

Sumida, T.; Murase, R.; Yoshimura, T.; Aramoto, T.; Ishikawa, A. \& Hamakawa, H. A case of impacted supernumerary forth molar in the bilateral mandiular ramus. Oral Sci. Int., 7(2):106-8, 2009.

Vaz, L. G. M.; Rodrigues, M. T. V. \& Ferreira Júnior, O. Cisto dentígero: características clínicas, radiográficas e critérios para o plano de tratamento. RGO, 58(1):12730, 2010.

White, S. C. Cone-beam imaging in dentistry. Health Phys., 95(5):628-37, 2008.

Yücel, O.; Yildirim, G.; Tosun, G.; Müge Baka, Z.; Bedii Göyenç, Y.; Günhan, O. Eruption of impacted permanent teeth after treatment of a dentigerous cyst: a case report. J. Dent. Child. (Chic.), 80(2):92-6, 2013.

Yulis, R.; Jiménez C.; Hernández, P. \& Ganai, A. Presentación inusual de un quiste dentigero en paciente pediátrico: Reporte de un caso y revisión de la literatura. Acta Odontol. Venez., 45(2):56-63, 2007.
Dirección para Correspondencia:

Rafael Binato Junqueira

Federal University of Juiz de Fora

Estado de Minas Gerais

BRAZIL

Email: binatojunqueira@gmail.com

Received: 12-09-2013

Accepted: 17-11-2013 